\title{
Analysis of Problem Oriented Systems Structure with Uncertainty Based on Fuzzy Graphs*
}

\author{
Bozhenyuk Alexander \\ Academy for Engineering and Technologies \\ Southern Federal University (SFEDU) \\ Rostov-on-Don, Russia \\ e-mail: avb002@yandex.ru
}

\author{
Ginis Larisa \\ Academy for Engineering and Technologies \\ Southern Federal University (SFEDU) \\ Rostov-on-Don, Russia \\ e-mail: gla@sfedu.ru
}

\begin{abstract}
It is offered to use the office of fuzzy graphs for modeling of problem oriented systems. The concepts of fuzzy transitive closure, fuzzy reciprocal transitive closure, and vitality degree of fuzzy graph are considered here. The algorithm for finding the vitality degree of fuzzy graph is described. Presented apparatus allows to analyze and to make decisions about the structure of the model of problem-oriented systems.
\end{abstract}

Keywords-problem oriented system, fuzzy model, fuzzy graph, vitality degree, fuzzy transitive closure, fuzzy reciprocal transitive closure

\section{INTRODUCTION}

The growing requirement of the accounting of a human factor (existence of uncertainty) when developing models for development of optimal administrative solutions in problem oriented systems proves relevance of the presented work.

Importance of research is caused including such factors as accumulation of new knowledge and data on modeling of complex systems [1]. It is necessary to emphasize that development of administrative decisions is a link of all main processes and functions proceeding in problem oriented systems as developed and introduced administrative decisions define not only efficiency of management process, but also a possibility of a sustainable development of the operated system and its robustness [2] in the conditions of the modern quickly changing world that dictates the accounting of uncertainty.

Unlike widespread approaches, for example, integration of multiagentny, imitating, evolutionary modeling or numerical methods [3], is offered as modern tools of modeling of problem oriented systems to use the office of fuzzy graphs [2] on the basis of the theory of systems and the theory of situational management.

\section{THEORETICAL ANALYSES}

In the course of studying of influence of external factors it is possible to allocate some accents for development of system. First, this is studying of possibility of identification of sensitivity of system to such influences, both at each level of hierarchy, and in a separate subsystem. Secondly, this is investigating the nature and character of such influences, their versions. And, in the third, this is researching of robustness of

*This work has been supported by the Russian Foundation for Basic Research, Project № 16-07-00336 system, including ability to resist to such influences.

Property robustness of problem oriented system can be considered as a certain average between such concepts as "reliability" and "safety". We will understand as robustness property of the system consisting in its ability to resist to influence of unforeseen external factors and to keep in advance set trajectory of development, ability of system to adapt for new situations, not stopping to "carry out" in full or in part the criterion function.

This property is certainly a complex integral one, which is assessed both quantitatively and qualitatively.

Problem oriented is the dynamic system intended for performance of a certain set of functions according to subject domain, which is characterized by hierarchical representation and existence of variables of various nature.

Any problem oriented system, in particular social and economic (SES) is the evolving object, developing under the influence of many changing factors, both internal, and external. By the nature structure SES is dynamical, and reflects evolution of system in time and space.

Classification of existing and most widespread approaches and the tools used at modeling and forecasting of functioning SES is brought in [4]. The idea of the hierarchical or so-called stratified description of system is put in a basis of modeling SES [5] and applied in [6].

Modeling on everyone stratum is offered to be conducted with use of the device of the directed graphs, which allows formalizing the expert knowledge. One of most important points of process of modeling is revealing of connections between objects, their orientation and influence.

Graphs models are most applicable to the systems, which problems ill-structured, and their basic advantage is possibility to be combined with other methods at different stages of research.

Graphs mathematical models, are intended for formalization of the description of complex object, a problem or functioning of system and revealing of relationships of cause and effect between their elements as a result of influence on these elements or changes of character of connections. These models are well suited to the following systems:

1) which are characterized by multi aspect processes 
occurring in them and their coherence;

2) which are characterized by absence of the sufficient quantitative information on dynamics of processes;

3) which are characterized by variability of processes in time, etc.

The list of these characteristics also defines complex and problem oriented system.

So, the model can be set using fuzzy directed graph. Fuzzy directed graph [7] is a pair of sets $\tilde{G}=(X, \tilde{U})$ in which $X=\left\{x_{i}\right\}$, $i \in I=\{1,2, \ldots, \mathrm{n}\}$ is a crisp set of vertices (or concepts), and $\tilde{U}=\left\{<\mu_{U}<x_{i}, x_{k}>/<x_{i}, x_{k}>>\right\}$ is a fuzzy set of edges (or arcs), where $<x_{i}, x_{k}>\in X^{2}$, and $\mu_{U}<x_{i}, x_{k}>$ is a degree of membership oriented edge $\left\langle x_{i}, x_{k}\right\rangle$ to fuzzy set of directed edges $\tilde{U}$.

The path of fuzzy graph $\tilde{l}\left(x_{i}, x_{j}\right)$ is called a direct sequence of fuzzy edges from vertex $x_{i}$ to vertex $x_{j}$, in which the final vertex of any edge is the first vertex of the following edge.

The conjunctive strength of the path of a fuzzy graph is defined by the formula:

$$
\mu_{\hat{\mathrm{I}}}\left(\mathrm{x}_{\mathrm{i}}, \mathrm{x}_{\mathrm{j}}\right)=\underset{<\mathrm{x}_{k}, \mathrm{x}_{\mathrm{i}}>\in \tilde{I}\left(\mathrm{x}_{\mathrm{i}}, \mathrm{x}_{\mathrm{j}}\right)}{\&} \mu_{\mathrm{U}}\left(\mathrm{x}_{\mathrm{k}}, \mathrm{x}_{\mathrm{t}}\right) .
$$

Fuzzy direct graph conveniently given in the form $\tilde{G}=(X, \tilde{\Gamma})$, where $X=\left\{x_{\mathrm{i}}\right\}, i \in I=\{1,2, \ldots, \mathrm{n}\}$, and $\tilde{\Gamma}$ - fuzzy valued mapping of the vertex set $X$ into itself, ie, $\tilde{\Gamma}: X \rightarrow X$, which is given in the form of fuzzy images of the elements $x \in X$, ie, $\tilde{\Gamma}\left(x_{i}\right)=\left\{<\mu_{\Gamma}\left(x_{j}\right) / x_{j}>\right\}, x_{j} \in \Gamma\left(x_{i}\right)$, here $\Gamma\left(x_{i}\right)$ is a crisp set of images of the vertices $x_{i} \in X$.

The vertices $X$ of graph $\tilde{G}=(X, \tilde{\Gamma})$ may be any objects of social, economic, political, ecological systems, or their combination, for example, a total national product and a financing of education, the purposes and necessary means of their achievement. The revealed connections between tops and its importance will be fuzzy arches.

Traditionally, there are two types of causality: the positive and negative in the graph model. Positive connection ("+" sign above the arc) means an increase in the value-factor causes and increases the value of the factor-investigation. Negative connection ("-" sign above the arc) means a reduction in value of the factor-causes and reduces the value of the factorinvestigation. Thus, the graph model can be set using the adjacency matrix $W=\left\{w_{i j}\right\}, w_{i j} \in\{-1,0,1\}$. However, the following is a more informative. In this paper we propose to transfer the accepted designation of force of connection on arches, from set: $\{$ ill connection $-\mathrm{W}$, average connection $\mathrm{M}$, strong connection $-\mathrm{S}\}$ in a value from an interval $[0,1]$. For example, 0 - connection is absent, [0.1, 0.3] - connection weak, $[0.4,0.6]$ - connection average, $[0.7,1]$ - connection strong. In our opinion it much more expediently also answers the requirement fuzzy graphs.

Besides, the three for concepts $\{-1,0,1\}$ which would designate value and importance of this or that top is offered. I.e., if the increase (or reduction) in one concept conducts to increase (or, to reduction) in other we appoint value $=1$. If there is no relation between concepts, it is assigned the value equal to 0 . If the increase (or reduction) in one concept conducts to reduction (or to increase) another, it is assigned the value equal to 1 .

Fuzzy graphs are widely used as models SES. The majority of isomorphic transformations of fuzzy graphs change their external representation. In this connection, definition invariants of fuzzy graphs are used. In particular, in papers $[8,9]$ the concepts of fuzzy bases and antibases are considered for the fuzzy graphs, and the method of their calculation is offered for the analysis of complex model as a whole, and in particular, for the decision of the research problem of sensitivity.

In paper [10] application of the device of fuzzy logic is described and possibility of use of the device of fuzzy sets is considered. The way of a finding of transitions between reference situations in the multilevel problem oriented system, based on comparison of fuzzy intervals is offered, the algorithm of comparison of fuzzy sets on an individual interval is in detail stated.

In paper [11] the approach using fuzzy sets for modeling of force of operating influence at different types of connections between the previous and subsequent purposes of functioning at various levels of hierarchy SES is offered. The choice of the administrative decision in problem oriented system by means of a method of the analysis of hierarchies is always accompanied by uncertainty. The situation when the weight over an arch of the fuzzy graph is designated by a fuzzy interval is considered. For search of decisions in a fuzzy hierarchical control system where separate tops are presented by fuzzy intervals with borders on different scales, the approach which is based on definition of degree of fuzzy equality of fuzzy numbers is inapplicable. Therefore it is offered to apply the approach based on comparison of fuzzy intervals.

The estimation and the analysis concern the same problems received graphs models from the point of view of their vitality. In the crisp graphs vitality is understood as its sensitivity to damages from the point of view of removal of some edges or vertices [12]. In case of fuzzy graphs, depending on tasks in view, under vitality different concepts, including degree of strong connectivity of the fuzzy graph [13, 14] can be understood.

\section{TECHNIQUE}

Let $\tilde{L}\left(x_{i}, x_{j}\right)$ be a family of the fuzzy graph paths from vertex $x_{i}$ to vertex $x_{j}$. Then the value $\tau\left(x_{i}, x_{j}\right)=\max _{i \in \bar{L}}\left\{\mu_{l}\left(x_{i}, x_{j}\right)\right\}$ defines the reachability degree of vertex $x_{j}$ for vertex $x_{i}$.

We will consider the degree of fuzzy graph vitality as a degree of strong connection, so it will be defined by the formula:

$$
V(\tilde{G})=\underset{x_{i} \in X}{\&} \underset{x_{j} \in X}{\&} \tau\left(\mathrm{x}_{\mathrm{i}}, \mathrm{x}_{\mathrm{j}}\right)
$$


It means there is a path between each pair of graph vertices with the conjunctive strength not less than value $V(\widetilde{G})$.

Let a fuzzy graph $\tilde{G}=(X, \tilde{U})$ is given. Let's define fuzzy multiple-valued reflections $\tilde{\Gamma}^{1}, \tilde{\Gamma}^{2}, \tilde{\Gamma}^{3}, \ldots, \tilde{\Gamma}^{k}[10]$ as:

$$
\tilde{\Gamma}^{1}\left(x_{i}\right)=\left\{<\mu_{\Gamma^{1}\left(x_{i}\right)}\left(x_{j}\right) /\left(x_{j}\right)>\right\},
$$

here $\left(\forall x_{j} \in X\right)\left[\mu_{\Gamma^{1}\left(x_{i}\right)}\left(x_{j}\right)=\mu_{U}<x_{i}, x_{j}>\right], \tilde{\Gamma}^{2}\left(x_{i}\right)=\tilde{\Gamma}\left\{\tilde{\Gamma}\left(x_{i}\right)\right\}$, $\tilde{\Gamma}^{3}\left(x_{i}\right)=\tilde{\Gamma}\left\{\tilde{\Gamma}^{2}\left(x_{i}\right)\right\}, \ldots, \quad \tilde{\Gamma}^{k}\left(x_{i}\right)=\tilde{\Gamma}\left\{\tilde{\Gamma}^{k-1}\left(x_{i}\right)\right\}=\left\{<\mu_{\Gamma^{k}\left(x_{j}\right)}\left(x_{j}\right) / x_{j}>\right\}$, here $\left(\forall x_{j} \in X\right)\left[\mu_{\Gamma^{k}\left(x_{i}\right)}\left(x_{j}\right)=\underset{\forall x_{i} \in X}{\vee} \mu_{\Gamma^{k-1}\left(x_{j}\right)}\left(x_{l}\right) \& \mu_{U}<x_{l}, x_{j}>\right]$.

It is obvious, that $\tilde{\Gamma}^{k}\left(x_{i}\right)$ is a fuzzy subset of vertices, which it is accessible to reach from $x_{i}$, using fuzzy ways of length $k$.

Fuzzy transitive closure $\tilde{\tilde{\Gamma}}\left(x_{i}\right)$ is fuzzy multiple-valued reflection:

$$
\tilde{\tilde{\Gamma}}\left(x_{i}\right)=\tilde{\Gamma}^{0}\left(x_{i}\right) \cup \tilde{\Gamma}\left(x_{i}\right) \cup \tilde{\Gamma}^{2}\left(x_{i}\right) \cup \ldots=\bigcup_{j=0}^{\infty} \tilde{\Gamma}^{j}\left(x_{i}\right)
$$

Here, by definition: $\tilde{\Gamma}^{0}\left(x_{i}\right)=\left\{<1 / x_{i}>\right\}$.

In other words, $\tilde{\tilde{\Gamma}}\left(x_{i}\right)$ is fuzzy subset of vertices, which it is accessible to reach from $x_{i}$ by some fuzzy way with the greatest possible conjunctive durability. As we consider final graphs, it is possible to put, that:

$$
\overline{\tilde{\Gamma}}\left(x_{i}\right)=\bigcup_{j=0}^{n-1} \tilde{\Gamma}^{j}\left(x_{i}\right)
$$
as [9]:

Similarly, we define the fuzzy reciprocal transitive closure

$$
\tilde{\widetilde{\Gamma}}^{-}\left(\mathrm{x}_{\mathrm{i}}\right)=\tilde{\Gamma}^{0}\left(\mathrm{x}_{\mathrm{i}}\right) \cup \tilde{\Gamma}^{-1}\left(\mathrm{x}_{\mathrm{i}}\right) \cup \tilde{\Gamma}^{-2}\left(\mathrm{x}_{\mathrm{i}}\right) \cup \ldots=\bigcup_{\mathrm{j}=0}^{\infty} \tilde{\Gamma}^{-\mathrm{j}}\left(\mathrm{x}_{\mathrm{i}}\right)
$$

In other words, $\tilde{\tilde{\Gamma}}^{-}\left(x_{i}\right)$ is fuzzy subset of vertices, from which it is accessible to reach vertex $x_{i}$ by some fuzzy way with the greatest possible conjunctive durability.

To find the vitality degree of a fuzzy graph we use the method of Malgrange to find the maximal strongly connected components of fuzzy graphs [7].

To do this, take an arbitrary vertex $x_{i} \in X$ and find for it fuzzy transitive closure and fuzzy reciprocal transitive closure, which are fuzzy sets on the vertex set $X$. Then we find their intersection:

$\tilde{\mathrm{C}}\left(\mathrm{x}_{\mathrm{i}}\right)=\tilde{\tilde{\Gamma}}\left(\mathrm{x}_{\mathrm{i}}\right) \cap \tilde{\tilde{\Gamma}}^{-}\left(\mathrm{x}_{\mathrm{i}}\right)=\left\{<\alpha_{1} / \mathrm{x}_{1}>,<\alpha_{2} / \mathrm{x}_{2}>, \ldots,<\alpha_{\mathrm{i}} / \mathrm{x}_{\mathrm{i}}>, \ldots,<\alpha_{\mathrm{n}} / \mathrm{x}_{\mathrm{n}}>\right\}$, where $\alpha_{j} \in[0,1]$.

If the carrier of fuzzy set $\tilde{C}\left(x_{i}\right)$ coincides with the set of vertices $X$, then the fuzzy graph $\tilde{G}=(X, \tilde{\Gamma})$ is strong connection, and the degree of its vitality will be defined as $V(\tilde{G})=\min \left\{\alpha_{1}, \alpha_{2}, \ldots, \alpha_{n}\right\}$. Otherwise the value $V=0$.

Now we will consider formal method allowing finding the degree of fuzzy graph vitality. Let fuzzy graph $\tilde{G}$ is presented by adjacent matrix $R_{X}=\left\|r_{i j}\right\|$ with dimensions $(n \times n)$.

We will consider four vectors-columns and four vectorslines with dimensions $(n \times 1)$ and $(1 \times n)$ accordingly:

Vector-column $L$ - length of a path (number of edges) from first vertex to considered vertex; Vector-column $X_{\text {prev }}$ of the previous vertices; Vector-column $\tilde{\tilde{\Gamma}}$ of fuzzy transitive closure; Vector-column $P_{\text {exam }}$ of the examined vertices; Vector-line $L^{-}$- length of a path (number of edges) from considered vertex to first vertex; Vector-line $X_{\text {prev }}^{-}$of the previous vertices; Vector-line $\tilde{\tilde{\Gamma}}^{-}$of fuzzy reciprocal transitive closure; Vector-line $P_{\text {exam }}^{-}$of the examined vertices.

An algorithm has a following view:

$1^{0}$. Give an initial values for all indices $x_{i} \in X, i=\overline{1, n}$ : $\tilde{\Gamma}\left(x_{i}\right)=0, \tilde{\Gamma}^{-}\left(x_{i}\right)=0, L\left(x_{i}\right)=\infty, L^{-}\left(x_{i}\right)=\infty, X_{\text {prev }}\left(x_{i}\right)=\varnothing$, $X_{\text {prev }}^{-}\left(x_{i}\right)=\varnothing, P_{\text {exam }}\left(x_{i}\right)="-", P_{\text {exam }}^{-}\left(x_{i}\right)="-"$. Go to step $2^{0}$.

/we define the fuzzy transitive closure of vertex $x_{1} /$.

$2^{0}$. Set $i:=1 ; L(i):=0$ (number of edges in the path from vertex $x_{1}$ to vertex $x_{1}$ ); $\tilde{\Gamma}(i):=1$ (reachability degree from $x_{1}$ to $x_{1}$ ). Go to step $3^{0}$.

$3^{0}$. If for all $x_{j}, j=\overline{1, n}, j \neq i: r_{i j}>\tilde{\Gamma}\left(x_{j}\right) \& \tilde{\Gamma}\left(x_{i}\right)>\tilde{\Gamma}\left(x_{j}\right)$, then set $\tilde{\Gamma}\left(x_{j}\right)=r_{j j} \& \tilde{\Gamma}\left(x_{i}\right)$, else $\tilde{\Gamma}\left(x_{j}\right)=r_{i j}$. And at the same time $L\left(x_{j}\right)=L\left(x_{i}\right)+1, \quad X_{\text {prev }}\left(x_{j}\right)=x_{i} \quad$ and $\quad P_{\text {exam }}\left(x_{i}\right)="+"$ (vertex $x_{i}$ was examined). Go to step $4^{0}$.

$4^{0}$. Determine such vertex $x_{k}$ of graph $\tilde{G}$, for it's index $k$ the conditions are $\tilde{\Gamma}\left(x_{k}\right)=\max _{\forall j=1, n, j i \neq}\left\{\tilde{\Gamma}\left(x_{j}\right) \mid L\left(x_{j}\right)<(n-1) \& P_{\text {exam }}\left(x_{j}\right)="-"\right\}$ (transitive closure of vertex more than transitive closure of the rest non examined vertices). Go to step $5^{0}$.

$5^{0}$. If such vertex $x_{k}$ exists, then we set $\mathrm{i}:=\mathrm{k}$ and go to step $3^{0}$, else go to step $6^{0}$. $x_{1} /$.

/we define the fuzzy reciprocal transitive closure of vertex

$6^{0}$. Set $i:=1 ; L^{-}(i):=0$ (number of edges in the path from vertex $x_{1}$ to $\left.x_{1}\right) ; \tilde{\tilde{\Gamma}}^{-}(i):=1$ (reachability degree from $x_{1}$ to $x_{1}$ ). Go to step $7^{0}$. 
$7^{0}$. If for all $x_{j}, \quad j=\overline{1, n}, j \neq i$ : $r_{i j}>\tilde{\Gamma}^{-}\left(x_{j}\right) \& \tilde{\Gamma}^{-}\left(x_{i}\right)>\tilde{\Gamma}^{-}\left(x_{j}\right)$, then set $\tilde{\Gamma}^{-}\left(x_{j}\right)=r_{j j} \& \tilde{\Gamma}^{-}\left(x_{i}\right)$, else $\tilde{\Gamma}^{-}\left(x_{j}\right)=r_{i j}$. And at the same time $L^{-}\left(x_{j}\right)=L^{-}\left(x_{i}\right)+1$, $X_{\text {prev }}^{-}\left(x_{j}\right)=x_{i}$ and $P_{\text {exam }}^{-}\left(x_{i}\right)="+"$ (vertex $x_{i}$ was examined). Go to step $8^{0}$.

$8^{0}$. Determine such vertex $x_{k}$ of fuzzy graph $\tilde{G}$, for it's index $k$ the conditions are $\tilde{\Gamma}^{-}\left(x_{k}\right)=\max _{\forall j=1, n, j i j}\left\{\tilde{\Gamma}^{-}\left(x_{j}\right) \mid L^{-}\left(x_{j}\right)<(n-1) \& P_{\text {exam }}^{-}\left(x_{j}\right)="-"\right\} \quad$ (inverse transitive closure of vertex more than inverse transitive closure of the rest non examined vertices). Go to step $9^{0}$.

$9^{0}$. If such vertex $x_{k}$ exists, then we set $\mathrm{i}:=\mathrm{k}$ and go back to step $7^{0}$, else go to step $10^{\circ}$.

$10^{0}$. Determine degree of fuzzy graph vitality $\widetilde{G}$ : $V(\tilde{G})=\min _{\forall i=1, n}\{\tilde{\tilde{\Gamma}}(i)\} \& \min _{\forall i=1, n}\left\{\tilde{\tilde{\Gamma}}^{-}(i)\right\}$. Go to step $11^{0}$.

$11^{0}$. End.

\section{Example.}

Let the fuzzy graph is given by adjacent matrix:

$A=\left[\begin{array}{ccccccccc} & 0,2 & 0,8 & & & & & & \\ 0,8 & & & 0,9 & & & & & \\ & & & & 0,7 & 0,2 & & & \\ 0,6 & & & & & & 0,5 & & \\ & & & 1,0 & & 0,6 & & & \\ & 0,6 & & & & & & 0,9 & \\ & & & & & & & & 0,6 \\ & & & 0,7 & & & & & 0,3\end{array}\right]$

For the considered graph fuzzy transitive closure has the form: $\tilde{\tilde{\Gamma}}\left(x_{1}\right)=\left\{\left\langle 1 / \mathrm{x}_{1}\right\rangle,\left\langle 0,6 / \mathrm{x}_{2}\right\rangle,\left\langle 0,8 / \mathrm{x}_{3}\right\rangle,\left\langle 0,7 / \mathrm{x}_{4}\right\rangle,\left\langle 0,7 / \mathrm{x}_{5}\right\rangle\right.$, $\left.\left\langle 0,6 / \mathrm{x}_{6}\right\rangle,\left\langle 0,5 / \mathrm{x}_{7}\right\rangle,\left\langle 0,6 / \mathrm{x}_{8}\right\rangle,\left\langle 0,5 / \mathrm{x}_{9}\right\rangle\right\}$, and fuzzy reciprocal transitive closure has the form: $\tilde{\Gamma}^{-}\left(x_{1}\right)=\left\{\left\langle 1 / \mathrm{x}_{1}\right\rangle,\left\langle 0,8 / \mathrm{x}_{2}\right\rangle\right.$, $\left\langle 0,6 / \mathrm{x}_{3}\right\rangle,\left\langle 0,6 / \mathrm{x}_{4}\right\rangle,\left\langle 0,6 / \mathrm{x}_{5}\right\rangle,\left\langle 0,6 / \mathrm{x}_{6}\right\rangle,\left\langle 0,6 / \mathrm{x}_{7}\right\rangle,\left\langle 0,6 / \mathrm{x}_{8}\right\rangle$, $\left.<0,5 / \mathrm{x}_{9}>\right\}$.

Further we find: $\tilde{C}\left(x_{1}\right)=\tilde{\Gamma}\left(x_{1}\right) \cap \tilde{\Gamma}^{-}\left(x_{1}\right)=\tilde{C}\left(x_{1}\right)=\tilde{\Gamma}\left(x_{1}\right) \cap \tilde{\Gamma}^{-}\left(x_{1}\right)$ $=\left\{\left\langle 1 / \mathrm{x}_{1}\right\rangle,\left\langle 0,6 / \mathrm{x}_{2}\right\rangle,\left\langle 0,6 / \mathrm{x}_{3}\right\rangle,\left\langle 0,6 / \mathrm{x}_{4}\right\rangle,\left\langle 0,6 / \mathrm{x}_{5}\right\rangle,\left\langle 0,6 / \mathrm{x}_{6}\right\rangle\right.$, $\left.\left\langle 0,5 / \mathrm{x}_{7}\right\rangle,\left\langle 0,6 / \mathrm{x}_{8}\right\rangle,\left\langle 0,5 / \mathrm{x}_{9}\right\rangle\right\}$.

Whence, the size of degree of vitality of the considered fuzzy graph equals 0.5 .

\section{CONCLUSION}

In conclusion it should be noted that the offered algorithm allows to allocate in problem oriented system the factors and communications between them which are earlier not considered in the existing models in view of the assumption of their insignificance, a neuchtennost and/or uncertainty.
Application of such approach to modeling on the basis of fuzzy graphs allows to develop reasonable administrative decisions for the purpose of definition of optimum ways of improvement of organizational structure of problem oriented system.

\section{REFERENCES}

[1] John H. Miller \& Scott, E.. Complex Adaptive Systems: An Introduction to Computational Models of Social Life. Published by Princeton University Press, 2007, 288p.

[2] A.V. Bozhenyuk, L.A. Ginis. Modeling and analysis of complex systems on the basis of fuzzy graph models. Life Science Journal. 2014. vol. 11, Spec. Is.7: 187-191.

[3] A.S. Antonova, K.A. Aksyonov. Multicriteria decision making under risk based on the integration of multi-agent, evolutionary modeling and numerical methods [Electronic resource] // Engineering journal of Don. - 2012 . № $4-2 . \quad 4 \quad-\quad$ URL: http://www.ivdon.ru/magazine/archive/n4p2y2012/1466

[4] L.A. Ginis. Scientific prediction in the modern world. TTI of SFEDU Press. 2011, 172p.

[5] M.D. Mesarovic, D. Macko and Y. Takahara, Theory of hierarchical, multilevel systems. Academic Press. New York and London. 1970. $344 p$.

[6] W.M. Haddad, V. Chellaboina and S. Nersesov. Impulsive and Hybrid Dynamical Systems: Stability, Dissipativity, and Control. Princeton University Press. 2006 pp.520.

[7] L. Bershtein, A. Bozhenyuk. Fuzzy graphs and fuzzy hypergraphs. Moscow Scientific world Press. 2005. 256p.

[8] L. Bershtein, A. Bozhenyuk, I. Rozenberg. Service Centers Finding by Fuzzy Antibases of Fuzzy Graph. In the Proceedings of SCAKD 2011 In Conjunction with the 4th International Conference on Pattern Recognition and Machine Intelligence, PReMI 2011, pp.12-22.

[9] L. Bershtein, A. Bozhenyuk, I. Rozenberg. Optimum allocation of centers in transportation networks by means of fuzzy graph bases. In the Proceedings of the 8th conference of the European Society for Fuzzy Logic and Technology (EUSFLAT-13), 2013. pp.230-235.

[10] L.A. Ginis, S.P. Vovk. Modeling of transitions among etalon situations in difficult systems in the conditions of uncertainty. Izvestiya SFedU. Engineering sciences, 2013. 2(139), pp.116-122.

[11] L.A. Ginis, S.P. Vovk. Modeling and forecasting of transitions between levels of hierarchies in difficult formalized systems. European Researcher, 2012. 20(5-1), pp.541-545.

[12] T. Starostina. Using component division criterion for fuzzy system vitality analysis. In the Proceedings 3 th conference of the European Society for Fuzzy Logic and Technology (EUSFLAT-03), 2003. pp. 785-788.

[13] A. Bozhenyuk, I. Rozenberg. Allocation of service centers in the GIS with the largest vitality degree. Communications in Computer and Information Science, 298 CCIS (Part 2). 2012. pp.98-106.

[14] A. Bozhenyuk, I. Rozenberg and D. Yastrebinskaya. Finding of service centers in GIS described by second kind fuzzy graphs. World Applied Sciences Journal, 2013. 22 (SPL.Issue2). pp.82-86. 
